\title{
Exploración del estado del arte en dispositivos inhibidores \\ de radio frecuencia ${ }^{1}$
}

\section{FAbio Alberto SuÁrez ${ }^{2,}{ }^{*}$ William AleXander CUEVAS ${ }^{3},{ }^{* *}$ JORGE ENRIQUE GAITÁN RUIZ ${ }^{4,}{ }^{* * *}$}

\section{Resumen}

Se ha venido realizando diferentes exploraciones en el Centro de Investigación (CEINV) de la Escuela de Comunicaciones Militares (ESCOM) ubicada en la ciudad de Facatativá (Cundinamarca), con el fin de desarrollar el diseño de un inhibidor o 'jammer' de drones, por medio de una onda moduladora con forma de señal triangular sumada a un ruido de tipo blanco con el fin, de que tenga las características de jamming de barrido, pues, en el estándar 802.11 es sensible a este tipo de interferencia, el cual es usado para el control de drones.

Cabe destacar, que existen diferentes estrategias de 'jamming' dependiendo la necesidad y las condiciones del entorno, donde, se emplearán las que se pueden definir en "jamming" por ruido de banda-ancha, "jamming" por ruido de banda parcial y "jamming" por ruido de banda-angosta, de las cuales, se derivan cuatro tipos de jammer basadas en las estrategias anteriormente mencionadas (Instituto Politécnico Nacional de México, 2001). Por lo tanto, se elegirá el más óptimo para cumplir con el objetivo de poder interrumpir la señal de control de un dron.

Palabras clave: drones, inhibidores, comunicación, seguridad nacional.

Clasificación JEL: L63, L64, L96, H56.
${ }^{1}$ Artículo de investigación. Escuela de Comunicaciones del Ejército, Colombia.

2 Ingeniero electrónico. Ingeniero de Producción y Operaciones, Universidad Pedagógica y Tecnológica de Colombia. Especialista en Seguridad Física y de Informática. Oficial de Innovación, ESCOM.

•fabio.suarez@escom.edu.co.

${ }^{3}$ Magíster en Ingeniería. Ingeniero electrónico,

Universidad Pedagógica y Tecnológica de Colombia. Asesor Centro de Investigación ESCOM.

"cienciatecnologia.escom@ cedoc.edu.co

${ }^{4}$ Estudiante del Semillero en Telecomunicaciones, ESCOM.

*** jorge.eg@gmail.com.

Fecha de recepción: 27 de enero de 2018 .

Fecha de aprobación: 9 de mayo de 2018.

Para citra este artículo: Mejía, E. y Molano, D. (2017) El terrorismo en la era de la información. Perspectivas en intgeligencia, 10(19): 289-298. 


\section{Abstract}

With the work that will be done during the internship at the Research Center (CEINV) of the School of Military Communications (ESCOM), located in the city of Facatativá (Cundinamarca), an inhibitor or 'jammer' of drones will be designed by means of a modulating wave in the form of a triangular signal added to a white noise in order to have the characteristics of jamming scanning, because in the 802.11 standard is sensitive to this type of interference, which is used for the control of drones.

It should be noted that there are different strategies of 'jamming' depending on the need and the conditions of the environment where it will be used; which can be defined in "jamming" by broadband noise, "jamming" by partial band noise and "jamming" by narrowband noise, from which four types of jammer are derived based on the aforementioned strategies [one]; of which the most optimal will be chosen to fulfill the objective of being able to interrupt the control signal of a drone.

Keywords: drones, inhibitors, communication, national security.

JEL classification: L63, L64, L96, H56. 


\section{Planteamiento del problema}

En la actualidad, el uso de drones que reciben el nombre técnico Unmanned Aerial Vehicle (UAV) o en español Vehículo Aéreo no Tripulado (VANT) por parte de la población civil se ha vuelto muy común en todas las regiones del mundo, aunque en principio eran de uso militar exclusivo.

Con respecto al uso de los drones, por la población civil se llega a un nuevo problema como la invasión de la privacidad, porque estos cuentan con cámaras de alta definición, donde, algunas son con trasmisión en tiempo real y les da a los usuarios la capacidad realizar fotografías, hacer reconocimiento de residencias privadas o militares y dando la posibilidad de un ataque terrorista, de contrabandear drogas y armas en cárceles por la fuerza que posee tiene la capacidad de poder transportar objetos. Debido a su pequeño tamaño logran pasar desapercibidos al ojo humano (Semana, s.f).

En cuanto a los dispositivos inhibidores o jammers, estos tuvieron su nacimiento en la segunda guerra mundial, para poder interrumpir las comunicaciones enemigas o para engañar a los pilotos de los aviones y hacer fallar las misiones; Pero, en la actualidad estos dispositivos son empleados para bloquear comunicaciones de GSM, WIFI entre otros; puesto que hay lugares, en donde, no son permitidas las comunicaciones inalámbricas como en cárceles, cines, salas de teatro, bibliotecas, o lugares de reuniones políticas.

Entonces, con respecto a los problemas mencionados anteriormente, se presenta un interrogante ¿cómo prevenir ataques con UAVS al cantón de comunicaciones de Facatativá y aumentar la seguridad del mismo? Entonces, para ello existe la siguiente solución: desarrollar un dispositivo jammer que bloquee drones que funcionan en la frecuencia de $2.4 \mathrm{GHz}$, el cual, permita proteger a la población y el derecho a su privacidad, por medio de dispositivos de RF lograr crear una señal de interferencia esperando que se logre bloquear su uso.

\section{Justificación}

En medio de la guerra fría, las grandes potencias requerían de poder conocer las posiciones y los próximos movimientos de los países enemigos, con el objetivo de estar un paso por delante, el cual, podía ser ganado si se lograba bloquear la comunicación entre las torres de control y los pilotos de aviones de reconocimiento o enviando información falsa con respecto a sus misiones, marcando el origen de un nuevo conjunto de dispositivos llamados jammer (Instituto Politécnico Nacional de México, 2001). 
En tiempos más recientes, se ha dado a conocer un nuevo vehículo llamado UAV el cual, en un principio fue un dispositivo militar, que migró rápidamente a uso civil, y donde tiene grandes aplicaciones en muchas áreas de trabajo, como agroindustria, ciencia, climatología, educación, ocio, seguridad, periodismo, logística, entre otros (HEMAV, s.f)

De igual manera, se logra ver que estos UAV cuentan con una gran cantidad de sensores y captadores de video e imagen de alta calidad, que en manos de personas inescrupulosas pueden perturbar la paz e invadir la privacidad de hogares y zonas privadas o restringidas para el público, Por tanto, con el desarrollo del jamming, se espera prevenir ataques con UAVs interfiriendo en la señal de radio control y de esta manera bloquear el mando del mismo, derribando la posible amenaza para ser investigada por los entes de seguridad e inteligencia del Ejército Nacional y de Defensa Nacional.

\section{Marco teórico}

Las redes inalámbricas permiten la conexión entre dispositivos sin tener que hacer uso de cables o de tener que estar viendo el punto de conexión directamente. Estas han dado solución a una necesidad de lograr interconectar los dispositivos, que en su mayoría son móviles, siendo capaces de ofrecer una gran velocidad de transmisión de datos, en donde, debido a su alta frecuencia pueden atravesar objetos sólidos.

\section{Wifi}

Esta tecnología, permite la interconexión inalámbrica entre dispositivos, dando la capacidad de poder conectarse a internet a través de una red inalámbrica de área local, esta tecnología cuenta con un estándar internacional la ISO 802.11, donde, especifica la frecuencia que opera, los canales y el ancho de banda, la cual define la velocidad de cada canal y así convirtiéndola en s tecnología usada por los drones o UAVs.

\section{Jamming o interferencia electrónica}

La interferencia Electrónica (electronic "jamming") es una forma de guerra electrónica, en las que se irradia señales de interferencia hacia el radar del enemigo. El bloqueo del receptor de señales de energía tiene dos técnicas principales que son: la técnica de ruido y la técnica de repetidor y los tres tipos de interferencia de ruido son: el spot (punto), sweep (barrido), y barrage (presa) (Instituto Politécnico Nacional de México, 2001). 
- Interferencia Spot (punto) se produce cuando un jammer, centra todo su poder en una sola frecuencia, si bien esto, podría deteriorar seriamente la capacidad de seguir en la continuidad de interferencia, en un radar con agilidad de frecuencia no se vería afectada, porque la mordaza sólo puede bloquear una repetición; pero, los inhibidores de múltiples, podrían interferir en un rango de frecuencias, esto consume una gran cantidad de recursos para tener algún efecto en una periodicidad de radar, y probablemente seguiría siendo ineficaz.

- Interferencia de barrido (sweep) es cuando, el poder completo de un jammer es pasando de una frecuencia a otra, si bien esto, tiene la ventaja de ser capaz de interferir frecuencias múltiples en una sucesión rápida, no afectan a todos al mismo tiempo y, por lo tanto, limita la eficacia de ese tipo de bloqueo, aunque, dependiendo de la comprobación de errores en el (los) dispositivo(s), esto puede hacer que una amplia gama de dispositivos sea realmente inútil.

- Interferencia de presa (barrage) es la interferencia de las frecuencias de forma simultánea por un jammer único, la ventaja es que, las frecuencias múltiples pueden ser interferidas al mismo tiempo, sin embargo, el efecto de interferencia puede ser limitado porque el jammer requiere extender toda su potencia entre estas, así serán más frecuencias son bloqueadas, con la menor eficacia en cada uno.

Sin embargo, estos tipos de jamming se aplican a diferentes estrategias, las cuales, son especifican la forma en que se pueden aplicar estas técnicas, usando diferentes niveles de potencia y dando diferentes eficiencias, pues cada una de las estrategias nombradas adelante tienen diferentes usos debido a su forma de bloquear las señales (Instituto Politécnico Nacional de México, 2001).

\section{1) “Jamming" por ruido}

La portadora emitida por el jammer, es modulada por una señal aleatoria de ruido. en donde este se introduce y puede ocupar ya sea, todo el ancho de banda empleado por la señal AJ, o simplemente una parte de él; los efectos serán distintos, pero, se debe de considerar que no siempre se necesita atacar todo el ancho de banda para interrumpir de manera eficiente la comunicación, y se divide en "jamming" por ruido de banda-ancha, "jamming" por ruido de banda parcial y "jamming" por ruido de banda-angosta (Instituto Politécnico Nacional de México, 2001).

\section{2) "Jamming" por ruido de banda-ancha o banda completa}

El ruido de banda ancha o BBN (Broadband noise) introduce energía, a través de todo el ancho del espectro de frecuencias en el que opere la aplicación de 
ruido blanco, a este tipo de "jamming" se le conoce también como "jamming" de banda completa y este tipo de "jamming" es aplicable a cualquier tipo de señal AJ. El nivel de potencia de "jamming" se denomina J0, y está medido en Watts/ Hertz. El principal limitante de este tipo, es que tiene un bajo J0, ya que, la potencia es esparcida en una parte amplia del espectro. Así mismo, El BBN "jamming" funciona elevando el nivel de ruido en el receptor, lo que ocasiona un decremento en la relación señal-a-ruido y la eficiencia de este tipo de "jamming" depende del nivel de potencia y por tanto de la distancia entre el jammer y el receptor (Instituto Politécnico Nacional de México, 2001).

\section{3) "Jamming" por ruido de banda-angosta}

Conocido como NBN (Narrowband noise) esta manera de generar jamming, introduce energía en solamente un canal, el ancho de banda de esta energía podría abarcar todo el canal o simplemente una parte de él; la diferencia radica en la potencia empleada y el espectro cubierto; la eficiencia de esta forma de jamming dependerá en parte, del conocimiento de la aplicación blanco, esto es, porque se debe de atacar el lugar exacto en el espectro en donde se encuentren los canales de interés, la potencia se puede canalizar toda a una pequeña parte del espectro, lo que representa una ventaja (Instituto Politécnico Nacional de México, 2001).

\section{4) “Jamming" por tonos}

Esta estrategia, consiste en colocar un solo tono (ST single-tone), o varios tonos (MT, multiple-tone), a lo largo del ancho de banda, donde, se encuentra la señal AJ; la eficiencia de esta técnica depende completamente del lugar en el espectro donde se coloquen los pulsos, es por eso, que se requiere estudiar la señal objetivo de manera cuidadosa.

Por otra parte, un sistema DSSS (Direct Sequence Spread Spectrum, espectro ensanchado por secuencia directo) es posible emplear single-tone jamming, para modificar el desplazamiento (offset) en los receptores y ocasionar que se sobrepase el nivel máximo de la señal, lo que produce, que no se pueda recibir la información. La relación entre la fase del tono emitido por el jammer y la fase de la señal es un parámetro importante, porque si se manda un solo tono, éste estará presente, ya sea en la frecuencia del cero o del uno y si se encuentra en la frecuencia del uno, entonces la fase representa un problema, ya que, si el tono no se encuentra en esa fase, no se podrá bloquear o interferir la transmisión del símbolo.

En lo anteriormente mencionado, se da a entender que, si el tono se encuentra en la frecuencia del cero, entonces podrá bloquear la transmisión al símbolo 
siempre y cuando la potencia sea adecuada sin depender de la fase. En un caso de MT si los tonos se colocan en canales continuos, el desempeño del jammer será teóricamente igual al desempeño de jamming por ruido de bandaparcial; debido a que, los tonos de colocan en canales continuos, se conoce a este particular caso de MT como "comb jamming", del que se produzca una correcta interferencia, dependerá en primer lugar de que el tono se coloque en una parte del espectro, en donde, exista un tono que represente un símbolo, en ese caso el JSR debe ser lo suficientemente alto.

En segundo lugar, dependerá de que, una vez que el tono del jammer esté en la frecuencia del tono del símbolo, la fase entre ellos sea igual. Este tipo de "jamming" es muy poco eficiente contra sistemas FH debido a que, depende de que la señal salte a la frecuencia en la cual se ha colocado el tono emitido por el jammer, es por eso que, si se utilizan tonos, estos deben estar barriendo una parte del espectro y no estar en una frecuencia específica; este es el caso de una estrategia de "jamming" posterior (Instituto Politécnico Nacional de México, 2001).

\subsection{Protocolo de comunicación wifi estándar $802.11 B$}

El IEEE 802.11b, conocido más familiarmente con su nombre de marca, Wi-Fi, es una de las redes inalámbricas más extendidas en todo el mundo, en donde, en la comunicación se utiliza un enlace directo desde el emisor hasta el receptor, esto conlleva a que, puede haber interferencias entre las señales y Para evitarlo se utilizan varios canales de transmisión, como por ejemplo: La banda de $2.4 \mathrm{GHz}$, la frecuencia a la que se emite la señal de WiFi, tiene 11 canales, de los cuales, el 1, el 6 y el 11 no se solapan entre ellos (García, 2014, s.p)

\subsection{Drones o UAV (Unmanned Aerial Vehicles)}

Estas, son aeronaves sin un piloto humano a bordo, teniendo en cuenta que el vuelo está controlado por el control remoto de un conductor desde el suelo, sin embargo, estos vehículos comercialmente usan la frecuencia libre de $2.4 \mathrm{GHz}$. El método típico de lanzamiento y recuperación de una aeronave no tripulada, es mediante la función de un sistema automático guiado por el GPS del dron o un operador externo en tierra quien controla su aterrizaje de forma segura, existen una variedad de formas, tamaños, configuraciones y características de UAV, esto hace que, cada uno tenga diferentes distancias de operación y tiempos de vuelo (XDRONES, s.f).

La mayoría de los drones comerciales, usan el protocolo 802,11 en la frecuencia de $2.4 \mathrm{GHz}$ el cual, se usa para controlar el vuelo de estos vehículos, en donde la 
distancia máxima de operación conocida por el dron de gama alta PHAMTOM PRO 4 es de 7 kk (PHAMTOM, s.f). Teniendo en cuenta, que todos los demás son inferiores a este.

\subsection{Descripción de la propuesta}

Tomando en cuenta la información anteriormente mencionada y las diferentes estrategias empleadas en el diseño de jamming, se quiere emplear un dispositivo jammer para la frecuencia de $2.4 \mathrm{GHz}$ con el cual, se pueda inhabilitar las comunicaciones de tipo WIFI.

Este dispositivo se puede emplear usando un SDR (en español, radio definida por software) ya que, presentan una ventaja esencial, porque se permite realizar el procesamiento de señales, como tal filtrar, mezclar y modular señales, entonces, de esta manera se puede reducir el tamaño de dispositivos y transmisión de radiofrecuencias. Se planea usar un dispositivo USRP (Universal Software Radio Peripheral) los cuales, son una gama de los SDR diseñadas y vendidas por Ettus Research y su empresa matriz, National Instruments, estos dispositivos, son usados en laboratorios de investigación, por universidades y aficionados, porque presentan una gran capacidad para diseñar cualquier tipo de transmisor de radiofrecuencia en el rango de frecuencias que poseen. De esta manera se puede obtener la señal deseada para bloquear la comunicación no deseada, en este caso la de los drones con su control de mando.

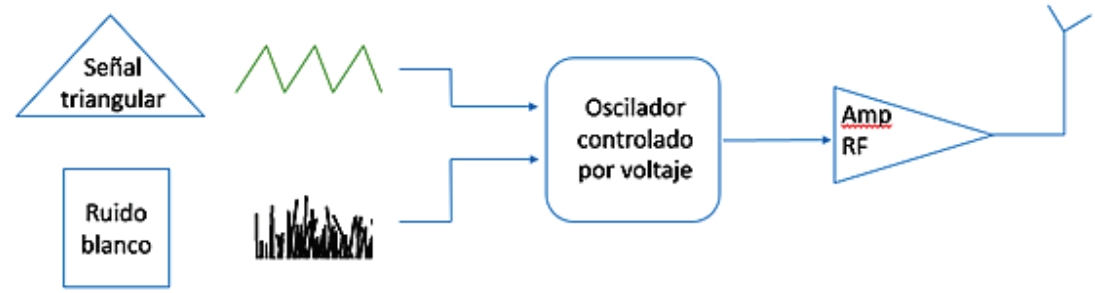

FIGURA 1. Proceso de señal jamming

Fuente: elaboración propia.

En la figura 1 allí se presenta el diagrama del diseño preliminar para la señal jamming, como una de las opciones que se plantea. Tomando en cuenta que el protocolo 802.11 es sensible en interferencias de señales triangulares, de tal manera, con esto se puede diseñar la señal jamming, es decir, sumando dos señales, siendo la primera triangular con una frecuencia de aproximadamente $110 \mathrm{KHz}$ y la segunda de ruido blanco, la cual, será la señal moduladora que una 
vez sumada, se procede a montar en la portadora de $2.4 \mathrm{GHz}$ y de esta manera poder u obtener una señal de RF que interfiera las señales de WIFI.

\section{Conclusiones}

Se observa que existen varios protocolos de comunicación inalámbrica, siendo las más conocidas las establecidas en la ISO 802.11, donde, la variedad de dispositivos en el mercado con capacidad de conectividad inalámbrica, cuentan con diferentes características en sus conexiones, en algunos casos usando más canales o más velocidad de transmisión; según las características de los dispositivos se tendrá en cuenta para el diseño de los jamming.

Así mismo, como hay diferentes métodos para transmitir información, o conectar inalámbricamente los dispositivos, existe gran variedad para usar las estrategias de jamming, todo dependiendo, del entorno y las condiciones que exigen, hay que estudiar de manera correcta el mejor método, en este caso para dispositivos en movimiento a larga distancia.

Hablando más claramente, sobre los dispositivos como tal, hay que tomar en cuenta la disponibilidad de Hardware y software, de la tal manera, que se logre diseñar un dispositivo que cumpla las necesidades, pues como se manejan frecuencias altas, los dispositivos que puedan ser programados o diseñados para que cumplan con suplir esta necesidad son escasos, ya sean SDR o los dispositivos análogos.

Debido a que, estos temas de inhibidores de frecuencias son censurados por el potencial, para generar una crisis de comunicaciones por su capacidad de interferir en el espectro electromagnético, no se consigue información muy detallada sobre el funcionamiento del mismo. 


\section{Referencias}

1. Instituto Politécnico Nacional de México. (2011). Diseño y elaboración de un jammer. Disponible de: http://tesis.ipn.mx/jspui/bitstream/123456789/9747/1/61.pdf.

2. Wikipedia. (s.f). Recuperado de: https://es.wikipedia.org/wiki/ Veh\%C3\%ADculo_a\%C3\%A9reo_no_tripulado .

3. Semana. (s.f). Recuperado de: http://www.semana.com/nacion/articulo/captan-envideo-a-contrabandistas-que-usaban-drones-para-llevar-droga-a-prision/550600.

4. HEMAV (s.f.). Las 5 principales de los drones para uso civil. Recuperado de: https:// hemav.com/las-5-principales-aplicaciones-de-los-drones-para-uso-civil/ .

5. E. T. S. D. I. Luis G. G. Diseño y análisis de un sistema de inhibición de frecuencia portátil en un entorno inalámbrico interior.

6. XDRONES (2016). Recuperadode:http://www.xdrones.es/wp-content/uploads/2016/07/ drone-jammer.pdf.

7. PHANTOM (s.f.). Recuperado de: https://www.dji.com/phantom-4-pro. 